5. Treggiari MM, Martin DP, Yanez ND, Caldwell E, et al. Effect of intensive care unit organizational model and structure on outcomes in patients with acute lung injury. Am J Respir Crit Care Med 2007;176(7):685-90.

6. Hixson ED, Davis S, Morris S, Harrison AM. Do weekends or evenings matter in a pediatricintensive care unit? Pediatr Crit Care Med 2005;6(5):523-30.

7. American Academy of Pediatrics Committee on Pediatric Emergency Medicine; American College of Emergency Physicians Pediatric Committee; Emergency Nurses Association Pediatric Committee. Joint policy statement-guidelines for care of children in theemergency department. Ann Emerg Med 2009;54(4):543-52.
8. American Academy of Pediatrics Committee on Pediatric Emergency Medicine, Frush K. Preparation for emergencies in the offices of pediatricians and pediatric primary care providers. Pediatrics 2007;120(1):200-12.

9. Santillanes G, Gausche-Hill M, Sosa B. Preparedness of selected pediatric offices to respond to critical emergencies in children. Pediatr Emerg Care 2006;22(11):694-8.

10. Jaimovich DG; Committee on Hospital Care and Section on Critical Care. Admission and discharge guidelines for the pediatric patient requiring intermediate care. Crit Care Med 2004;32(5):1215-8.

11. ThiriezG,Lefebvre A. Pediatric intermediate care demand, results of a survey in 3 French regions. Arch Pediatr 2010;17(8):1147-52.

\title{
Actualización del consenso sobre constancia de salud del niño y del adolescente para la realización de actividades físicas y/o deportivas

\author{
Guidelines on children preparticipation physical evaluation. Update
}

\begin{abstract}
Comité Nacional de Medicina del Deporte Infantojuvenil ${ }^{a}$, Grupo de Trabajo de Salud Escolar ${ }^{b}$,

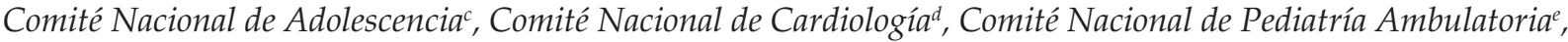
Comité de Medicina del Deporte Infantojuvenil filial Córdobaf y Servicio de Traumatología del Hospital de Niños Ricardo Gutiérrez ${ }^{8}$
\end{abstract}

\section{RESUMEN}

La práctica de actividades físicas en niños y adolescentes requiere que el pediatra realice un examen médico y una constancia que los habilite a participar.

Escuchandolainquietud de pediatras y entidades deportivas, nuestro Comité realizó una actualización sobre el tema.

Los puntos relevantes que tomamosencuenta para actualizar son el cambio del término "certificado" por el de "constancia"; la responsabilidad compartida entre el médico, los padres, la institución y los profesores a la hora de tener en cuenta al niño que hace actividad física; la determinación del momento ideal para pedir un electrocardiograma, y el énfasis sobre el aparato locomotor en el momento del examen físico.

La idea es que este modelo de ficha médica sea usado por las instituciones deportivas, escolares, etc., a nivel nacional para así poder aunar conceptos y desterrar el sinnúmero de fichas de salud que están dispersas por todo el país.

\section{ABSTRACT}

The practice of physical activities in children and adolescents requires that the pediatrician carry out a physical examination and extend certification that enables them to participate.

Correspondencia:

Dra. María Guinot: mguinot@intramed.net

Conflicto de intereses: Ninguno que declarar.

Recibido: 5-6-2013

Aceptado: 26-3-2014
When listening to doctors and sports institutions concerns, our Committee decided to update the topic. The relevant point that we have considered is to change the term "Certificate" for "Record", which implies shared responsibility among doctors, parents, institutionsand teachers when takingintoconsideration thechild that practices physical activity; the best time to request an electrocardiogram, and to emphasize attention on the locomotive apparatus when doing a physical examination. The idea is that this model of medical record will be used by educational and sports organizations at a national level in order tounifyconcepts and criteriaandleaveasidethecountlesshealth cards that are scattered throughout the country.

http:/ /dx.doi.org/10.5546/aap.2014.384

a. Dra. Laura Gaete, Dra. Elsa Galindo, Dra. Stella Maris Gil, Dra. María Guinot, Dra. Dora Haag, Dra. Patricia Jáuregui, Dra. Daniela Pacheco Agrelo, Dra. Juliana Pochetti,

Dra. Paula Rojas Filartiga, Dr. Leonardo Spada, Dra. Nelly Testa, Dr. Ángel Turganti.

b. Dra. Alicia Lukin, Dra. Graciela Mandolesi.

c. Dra. Mirta Garategaray, Dra. Patricia Goddard, Dra. Graciela Morales, Dra. Valeria Mulli.

d. Dra. Cintia Croxato, Dra. Dora Haag.

e. Dra. Fanny Breitman, Dr. Mario Elmo.

f. Dr. Oscar Casasnovas, Dr. Raúl Ferrari.

g. Dr. Marcelo Blanco, Dr. Juan C. Krauthamer. 


\section{INTRODUCCIÓN}

En las últimas décadas, se incrementó la promoción de la actividad física con la intención de favorecer su adherencia desde temprana edad y desarrollar habilidades y beneficios para la salud a lo largo de la vida. Esta incorporación desde el inicio escolar en actividades libres o en deportes organizados requiere que el pediatra realice un examen médico y una constancia que los habilite para participar.

Los niños/adolescentes inscriptos en deportes federados o que participan en clubes, por lo general, cumplen con este examen, pero no siempre se solicita cuando las competencias deportivas son independientes o libres.

A pesar de las controversias que ha generado este tipo de examen médico en cuanto a su utilidad para prevenir la morbilidad y la mortalidad en los niños y adolescentes que hacen deporte, sigue siendo una valiosa alternativa para un examen médico anual, actualización de inmunizaciones, identificación y manejo de condiciones crónicas de salud y un momento propicio para orientar sobre la prevención de lesiones y recomendaciones nutricionales.

Atento a la inquietud de los pediatras y de distintas entidades deportivas, el Comité Nacional de Medicina del Deporte Infantojuvenil trabajó junto con otros expertos en la actualización de un consenso para integrar todos los aspectos de la evaluación de salud del niño y del adolescente que realizan actividades físicas y/o deportivas, y confeccionar una ficha médica que pueda ser utilizada como patrón por las instituciones educativas y deportivas a la hora de extender una constancia que los habilite para la práctica de dichas actividades.

El propósito primordial de este examen médico es observar la salud del paciente y el impacto del entrenamiento sobre ella, y determinar si está en condiciones de realizar un esfuerzo físico.

Así, cuando extendemos una constancia para la práctica de actividades físicas/deportivas, el examen médico está orientado a:

- Detectar condiciones físicas que puedan predisponer una lesión con el ejercicio.

- Detectar condiciones que puedan poner en riesgo su vida ante la demanda de un esfuerzo físico.

- Cumplimentar los aspectos legales requeridos para la participación en actividades físicas.

La falta de consenso en el interrogatorio y en el examen físico lleva a que todos los años, al iniciar el ciclo escolar, los pediatras nos encontremos con distintos modelos de fichas médicas y órdenes de exámenes complementarios de las escuelas o instituciones deportivas que, por exceso u omisión, confunden la práctica y condicionan la realización de la constancia final. De esta manera, puede descalificarse totalmente al niño/adolescente para la actividad física o, por el contrario, autorizarlo sin el asesoramiento adecuado para prevenir complicaciones.

Es fundamental, entonces, cumplir los pasos previos a la extensión de la constancia y, en el caso de un hallazgo clínico, hacer las consideraciones necesarias a fin de lograr una participación más segura.

\section{METODOLOGÍA}

Se realizó la revisión de los consensos anteriores de la Sociedad Argentina de Pediatría (SAP) relacionados con este tema; a saber:

- Consenso sobre examen físico del niño y del adolescente que practican actividades físicas, publicado en el año 2000. ${ }^{1}$

- Consenso sobre certificación para la actividad física escolar y preparticipativa deportiva, publicado en el año 2001.2

- Actualización bibliográfica de los últimos diez años mediante la consulta de las bases de Pubmed y Medline sobre examen físico para la preparticipación deportiva.

- Revisión bibliográfica, tanto a nivel nacional como internacional, contemplando especialmente los lineamientos del consenso de Examen físico preparticipación deportiva (EFPD) de las sociedades americanas, italiana y de Brasil..$^{3-6}$

Para la definición de la ficha médica, se revisaron las utilizadas por el Centro de Estudios de Alto Rendimiento Deportivo (Cenard); el Examen Médico para la Mediana y Alta Competencia (EMMAC), Córdoba; la del Polideportivo de Almirante Brown; la de la Unión Argentina de Rugby (UAR); la de la Asociación Nacional de Hockey, y las diseñadas para los Torneos Bonaerenses y Salud Escolar del Gobierno de la Ciudad de Buenos Aires.

Para tal fin, se invitó a trabajar y se consultó al Comité de Medicina del Deporte InfantoJuvenil de la filial Córdoba, al Comité Nacional de Adolescencia, al Comité Nacional de Pediatría Ambulatoria, al Comité Nacional de Cardiología, al Grupo de Trabajo de Salud Escolar de la SAP y a integrantes del Servicio de Traumatología del Hospital de Niños Ricardo Gutiérrez. 
Se desarrolló un modelo de ficha médica a fin de que el pediatra encontrara en ella los elementos básicos que tiene que tener al momento de satisfacer la consulta y se prestó especial atención al redactar la constancia escrita (reemplaza al término "certificado") que se entrega al paciente y se presenta ante las diferentes instituciones escolares o deportivas.

\section{OBJETIVO PRIMARIO}

- Diseñar un modelo de ficha y de constancia para realizar la evaluación de salud del niño y del adolescente que participarán en actividades físicas y/o deportivas.

\section{OBJETIVOS SECUNDARIOS}

- Detectar precozmente la presencia de patología cardíaca.

- Disminuir los riesgos de padecer problemas o lesiones originados por la práctica de actividades físicas y /o deportivas.

- Alentar, a través de esta consulta, la promoción de hábitos saludables.

\section{FICHA MÉDICA}

La ficha médica propuesta consta de: 1) anamnesis y 2) examen físico.

La anamnesis está orientada a obtener información general sobre los antecedentes personales y familiares, poniendo especial atención en los aspectos de los sistemas músculoesquelético y cardiovascular, los más demandados frente a un esfuerzo físico y que pueden poner en riesgo la salud del niño o del adolescente que hace una actividad física o deportiva.

\section{Anamnesis}

La anamnesis es similar a la realizada en una consulta médica típica de control del niño o del adolescente, con especial énfasis en los antecedentes cardiovasculares y osteoarticulares.

Tomando como modelo distintas fichas médicas, se consensuó el modelo presentado en el Anexo 1.

Los aspectos interrogados representan algunos de los desafíos más comunes para el pediatra al momento del examen. Es muy importante explorar antecedentes personales, quirúrgicos, de enfermedades, traumatológicos y antecedentes familiares, con especial atención en los cardiovasculares.

La anamnesis reviste tal importancia que no debería examinarse ningún niño o adolescente que no concurriera a la consulta con la ficha de antecedentes completa.

La ficha de an a mnesis podrá ser autoadministrada; si el paciente es mayor de 14 años y hasta los 18 años se recomienda que la ficha de anamnesis sea completada por un adulto responsable. En caso que el adolescente concurra solo a la consulta, él mismo podrá completar la mencionada ficha. El médico evaluará la necesidad de ampliar los datos convocando a la familia o al adulto responsable de acuerdo al conocimiento previo de los antecedentes del paciente y su familia, al grado de madurez y la veracidad de las respuestas del adolescente considerando la autonomía progresiva de esta etapa de la vida (Ley $\mathrm{N}^{\mathrm{O}} 26061$. Ley de protección integral de los derechos de las niñas, niños y adolescentes) (ver Anexo 1).

Una vez evaluados los datos consignados en la mencionada ficha, se ampliará la anamnesis para relevar otros datos de importancia. En el caso de los adolescentes, la anamnesis personalizada se realizará en la entrevista a solas. Por tal motivo se decidió que formara parte de la ficha de examen físico. (Ley 26529. Derechos del paciente en su relación con los profesionales y las instituciones de la salud).

Luego se procederá a realizar el examen físico, consignando en la ficha los datos relevados (ver Anexo 2).

La ficha de anamnesis constituye un documento que junto con la ficha de examen físico del paciente deberá permanecer archivado en la historia clínica del mismo bajo la guarda del médico. Al paciente se le entregará la constancia para ser presentada ante la institución que así lo requiera.

\section{MODELO DE CONSTANCIA}

La constancia se extenderá solo luego de cumplimentar la anamnesis y el examen físico.

Finalizado el examen físico, se procederá a decidir si el niño/adolescente está en condiciones de realizar actividades físicas / deportivas.

La constancia es una responsabilidad compartida entre el adolescente y/o adulto responsables, el médico, los profesores de educación física y/o los entrenadores a cargo.

El adolescente y/o adulto que completa la anamnesis es responsable de la veracidad de los datos aportados en la misma, como así también del cumplimiento de las indicaciones médicas y de los controles. 
El médico se responsabilizará de la valoración clínica. Los responsables de la institución deportiva, de lascondiciones de seguridad medioambiental y de la idoneidad del personal a cargo del niño/adolescente.

La constancia puede ser manuscrita o impresa.

En el apartado "Observaciones", se detallarán los hallazgos del examen físico.

Se presenta un modelo de constancia: Figura 1.

\section{CONSIDERACIONES ESPECIALES}

\section{Antropometría}

Para la valoración antropométrica, se utilizarán el peso y la talla según edad y sexo e índice de masa corporal (IMC: peso $/$ talla $^{2}$ ) con sus respectivos percentiles, según tablas de la Organización Mundial de la Salud (OMS).

Hay que tener en cuenta, a la hora de interpretar el IMC, que algunos deportistas adolescentes pueden superar el percentilo 85 del IMC por aumento de masa ósea, muscular y el volumen plasmático como consecuencia del entrenamiento en ciertas disciplinas deportivas (ejemplo: rugby).

\section{Evaluación cardiovascular}

La muerte súbita (MS), aunque rara en esta población (Maron y col., 1/200 000 casos por año; Van Camp y col., 1/133 000 casos en varones y $1 / 769000$ casos en mujeres), es siempre trágica e impactante, con los consabidos aspectos legales y médicos. ${ }^{78} \mathrm{Si}$ bien las causas más frecuentes son las cardíacas (80-90\% de los casos), debemos tener presente el consumo de sustancias al momento de hacer la anamnesis, ya sean drogas de abuso (cocaína, efedrina, anfetaminas) o medicamentos utilizados en el tratamiento de enfermedades (salbutamol, clembuterol, insulina), el golpe de calor y la deshidratación como otras causas potenciales de muerte súbita.

En la Tabla 1, se resumen las principales causas de muerte súbita de origen cardíaco relacionadas con el ejercicio. ${ }^{9-13}$

En la anamnesis, se puede detectar y/o sospechar la mayoría de los factores de riesgo cardiovasculares para la práctica deportiva.

- Antecedentes familiares: muerte súbita en familiares jóvenes (menores de 50 años), miocardiopatías, arritmias, anomalías cardíacas congénitas, hipertensión arterial, diabetes, hipercolesterolemia. ${ }^{14}$

- Antecedentes personales: cardiopatías congénitas o adquiridas detectadas (miocarditis, compromiso coronario por enfermedad de Kawasaki, etc.), hipertensión arterial, síncope o presíncopes con esfuerzo (sugiere causa cardíaca, es potencialmente fatal y siempre requiere una evaluación y tratamiento especializados), arritmias, dolor torácico, palpitaciones, disnea de esfuerzo, internaciones previas, intervenciones quirúrgicas.

En el examen clínico, serán relevantes los siguientes datos: 15,16

- Inspección: aspectogeneral en busca de estigmas genéticos (síndrome de Marfan).

- Palpación: pulsos humerales y femorales, hepatomegalia, búsqueda de frémito en hueco supraesternal, choque de la punta.

- Auscultación: precordio, base, axilas y dorso. Determinación de características de los ruidos, presencia de soplos o ruidos agregados.

- Control de tensión arterial.

Figura 1. Modelo de constancia

Dejo constancia que .................................., DNI: . ., de.......... años de edad ha sido evaluado clínicamente y se encuentra en condiciones de salud para realizar actividades físicas y deportivas acordes a su edad, sexo, estadio madurativo y bajo supervisión de personal idóneo.

Observaciones: Fecha
Fen......................
Firma y sello del médico

Tabla 1. Causas de muerte súbita con el ejercicio

- Miocardiopatías (hipertrófica, dilatada o no compacta).

- Displasia arritmogénica de ventrículo derecho.

- Alteraciones eléctricas (síndrome de QT prolongado, taquicardia ventricular polimorfa catecolaminérgica, síndrome de Brugada, síndromes de preexcitación, bloqueos AV, síndrome de QT corto).

- Síndromes genéticos (síndrome de Marfan).

- Anomalías estructurales (anomalía de implantación coronaria, obstrucciones al tracto de salida del ventrículo izquierdo). 


\section{Electrocardiograma}

Existe controversia entre las diferentes asociaciones relacionadas con la actividad física de niños y adolescentes, tanto a nivel nacional como internacional, sobre la indicación y el momento ideal para la realización de un electrocardiograma (ECG) en reposo para la práctica deportiva como método de pesquisa para prevenir la MS.

Mientras la Asociación Estadounidense de Cardiología no lo incluye en sus recomendaciones y basa toda la pesquisa cardíaca en la historia clínica y en la exploración física, la Sociedad Europea de Cardiología y el Comité Olímpico Internacional adoptan la experiencia de Italia, que, hace más de treinta años, promovió una legislación muy concreta sobre los exámenes preparticipación deportiva, con obligatoriedad de realizar ECG en reposo. ${ }^{3,4}$ En nuestro país, no hay datos estadísticos que estimen la incidencia de MS en esta población.

Los niños sanos, sin antecedentes familiares ni personales patológicos y sin intercurrencias con afectación cardiovascular, no requieren ECG para la realización de actividad física. 5,17-20

En la adolescencia, existe mayor riesgo de arritmias por miocardiopatías y trastornos eléctricos por los cambios hormonales ocurridos en el empuje puberal. Por tal motivo, en esta etapa, se recomienda hacer un ECG si el paciente no tiene uno previamente realizado y con resultado normal, junto con el interrogatorio y el examen físico.

El ECG informado como normal no tiene indicación de ser repetido, excepto que el niño/ adolescente presente patología adquirida con repercusión cardiovascular.

Los estudios de mayor complejidad, como el ecocardiograma y la ergometría, deberán estar a cargo del especialista. Esta última puede indicarse para detectar factores de riesgo durante el esfuerzo (hipertensión arterial, arritmias), conocer la capacidad funcional y el grado de entrenamiento del individuo, en especial en los que realizan deportes competitivos y en los portadores de cardiopatía congénita corregida o no, miocardiopatías, valvulopatías y frente a síntomas como palpitaciones, disnea, cianosis, precordialgia típica o atípica, síncope con el esfuerzo.

\section{Examen músculo-esquelético}

El aparato locomotor del niño está sometido a constantes cambios, producto del crecimiento y del desarrollo. En comparación con el esqueleto relativamente estático de los adultos, existen importantes diferencias morfológicas, fisiológicas y biomecánicas propias de su inmadurez que pueden exponerlo a diversas alteraciones frecuentemente encontradas en los exámenes deportivos.

La anamnesis acerca de las lesiones sufridas durante el año, que documenta cuáles fueron los diagnósticos finales y los tratamientos correspondientes, reviste fundamental importancia a fin de evitar la subestimación de las lesiones deportivas. La reiteración de una lesión o la falta de un tratamiento precoz pueden acarrear una consecuencia más grave, deserciones precoces y patología degenerativa a edades tempranas.

Las bases del examen músculo-esquelético serán las mismas que las consideradas en el consenso anterior, y se pondrá especial atención en la actitud y la postura, simetrías corporales, movilidad articular y evaluación de la fuerza.

Un concepto para tener en cuenta es equiparar las categorías deportivas, sobre todo las infantiles, según edades biológicas más que cronológicas, principalmente en los deportes que requieran contacto. En la práctica diaria, este concepto no se tiene en cuenta, y hay diferencias de envergadura, masa ósea y muscular, fuerza y potencia, etc., a igual edad cronológica. Esto pone en una situación de mayor vulnerabilidad a quienes están en percentilos menores por su crecimiento biológico, que tienen mayor predisposición para sufrir lesiones.

Con los aspectos generales descritos anteriormente, pretendemos desarrollar un examen deportivo que nuclee los conceptos preventivos, legales y epidemiológicos, como así también el seguimiento, a fin de disminuir riesgos generados por diversos factores que pueden condicionar la práctica deportiva a futuro. ${ }^{21-24}$

\section{CONDICIONES FÍSICAS QUE LIMITAN LA PARTICIPACIÓN DEPORTIVA}

Existen condiciones físicas que requieren restricción o exclusión de la participación deportiva. Para la mayoría de las enfermedades crónicas, la evidencia científica permite la participación deportiva.

El momento d e decidir si ese niño o adolescente con determinada condición física o enfermedad puede hacer deporte es siempre complejo y deben tenerse en cuenta las recomendaciones especiales inherentes a la enfermedad en cuestión. 
Al pediatra se le presentan varios interrogantes y tendrá que evaluar lo siguiente:

- Si el hallazgo puede predisponer a una lesión.

- Si el hallazgo pone en riesgo a los compañeros de equipo u otros participantes.

- Si el paciente puede participar igual cumpliendo el tratamiento específico (medicación, rehabilitación, vendajes).

- Si debería elegir otra actividad mientras está completando el tratamiento, otra posición en el campo, etc.

En caso de que por su condición física no pudiera realizar determinadas actividades, debemos orientarlo siempre hacia otras para evitar el sedentarismo (véase la clasificación de deportes en la Tabla 2).

Si el hallazgo es de origen cardiovascular, es el cardiólogo quien determinará la exclusión o participación y podrá consultar las recomendaciones para los atletas con anomalías cardiovasculares, surgidas de la $36^{\text {a }}$ Conferencia de Bethesda. ${ }^{25}$

Existen tres condiciones que contraindican absolutamente la participación en una actividad física, dado que ponen en riesgo la vida y son:

- Carditis: puede condicionar una muerte súbita.

- Fiebre: puede indicar un proceso patológico en curso (infeccioso o no), que aumenta el metabolismo y la frecuencia cardíaca. Del mismo modo, puede almacenarse mucho calor, disminuir la tolerancia a altas temperaturas, predisponer una enfermedad por calor, aumentar el esfuerzo cardiopulmonar, disminuir la capacidad máxima de ejercicio y aumentar el riesgo de hipotensión por alteración del tono vascular y deshidratación. En ocasiones, la fiebre acompaña una miocarditis u otras enfermedades que pueden poner en riesgo la vida.

- Diarrea:puede incrementar el riesgo de deshidratación y una enfermedad por calor.

Otras condiciones médicas requieren la suspensión de la actividad según la etapa de la enfermedad y el estado del paciente. Algunas recomendaciones específicas sobre la participación de pacientes con patología crónica pueden consultarse en el Anexo 3, actualizado y publicado en el año 2008 por la Academia Estadounidense de Pediatría. A pesar de las aclaraciones referidas para cada patología, consideramos que, dada la complejidad de ciertos casos, se debe hacer siempre una evaluación individual y quedará a criterio del pediatra consultar con un especialista, con un apropiado conocimiento para asesorar al niño y a la familia.

\section{CONCLUSIÓN}

$\mathrm{L}$ a actividad física es fundamental en el desarrollo infantil y para adquirir un estilo de vida saludable. Existen pocas contraindicaciones absolutas que justifiquen la suspensión de la actividad física.

TABLA 2. Clasificación de los deportes por contacto

\begin{tabular}{|c|c|c|}
\hline Contacto & Contacto limitado & Sin contacto \\
\hline Baloncesto & Carreras de aventura & Bádminton \\
\hline Boxeo & Béisbol & Culturismo \\
\hline Saltos de trampolín & Ciclismo & Bolos \\
\hline Deportes extremos & Canoas o kayak (aguas bravas) & Canoas o kayak (aguas mansas) \\
\hline Hockey sobre césped & Esgrima & Remo \\
\hline Fútbol americano & Atletismo en pista, salto de altura, & Baile \\
\hline Gimnasia & salto con pértiga & Atletismo en pista \\
\hline Hockey sobre hielo & Equitación & Lanzamiento de disco \\
\hline Artes marciales & Artes marciales & Lanzamiento de jabalina \\
\hline Rodeo & Ráquetbol & Lanzamiento de peso \\
\hline Rugby & Patinaje (sobre hielo, en línea, & Golf \\
\hline Esquí, descenso & convencional) & Marcha atlética \\
\hline Saltos de esquí & Esquí (de fondo, acuático) & Tiro al blanco \\
\hline Esquí con tabla & Squash & Salto de comba \\
\hline Fútbol & Voleibol & Carrera \\
\hline Hándbol & Halterofilia & Vela \\
\hline Disco volador & Windsurf o surf & Buceo \\
\hline Polo acuático & & Natación \\
\hline \multirow[t]{3}{*}{ Lucha libre } & & Tenis de mesa \\
\hline & & Tenis \\
\hline & & Senderismo \\
\hline
\end{tabular}


La consulta para obtener la constancia de actividad física (ya sea en la escuela o en una entidad deportiva) es una valiosa oportunidad para hacer prevención en salud, máxime en la adolescencia, dado que suele ser la única visita para control médico.

La presencia de antecedentes familiares o personales de enfermedad cardiovascular surgidos en el interrogatorio, aun frente a un examen físico normal, requiere una evaluación cardiológica. Los resultados de dicha evaluación y las recomendaciones sugeridas por el especialista deben constar en la ficha médica y, en lo posible, se debe adjuntar el informe.

Debemos hacer hincapié en hábitos que favorezcan el buen desempeño deportivo, como ser alimentación saludable, horas de sueño, una adecuada hidratación y protección solar, y aconsejar sobre el uso del equipamiento protector para las distintas disciplinas deportivas (ejemplo: protector bucal, testicular, rodilleras, casco, gafas, etc.). En los adolescentes, se reforzará la prevención del uso de anabólicos, ergogénicos, suplementos dietarios u otras sustancias, especialmente en varones; en las mujeres, sobre hábitos alimenticios y ciclos menstruales, y se buscarán trastornos de la conducta alimentaria. Además, se investigará sobre el consumo de alcohol, tabaco y drogas.

Sugerimos que aquellos adolescentes que desempeñan actividades con grandes exigencias físicas (entrenamientos semanales, competiciones los fines de semana más las actividades escolares) sean evaluados al menos dos veces en el año por el pediatra a fin de vigilar los efectos del entrenamiento en el proceso de crecimiento y desarrollo, y pesquisar situaciones (lesiones minimizadas por el paciente o sus padres, presiones psíquicas o físicas) que pongan en riesgo su bienestar o la continuidad de la actividad, ya que esta es la edad de mayor abandono deportivo.

Es nuestro objetivo como médicos de cabecera de niños y adolescentes seguir el control de nuestros pacientes en todos sus aspectos, con el consejo anticipado para la prevención de lesiones tanto físicas como psíquicas en pos de un desarrollo integral hasta la edad adulta.

\section{Agradecimientos}

Al Dr. Juan Carlos Ferrerías y al Dr. Carlos Escudero por el asesoramiento legal.

\section{GLOSARIO}

- Actividad física: es cualquier movimiento corporal producido por los músculos esqueléticos y que provoca un gasto energético por encima de la tasa de metabolismo basal (OMS). Incluye actividades de la rutina diaria, como las tareas del hogar, ir de compras, trabajar.

- Ejercicio físico: es la actividad física planeada, estructurada, repetitiva y que persigue un propósito de entrenamiento para mejorar o mantener uno o más componentes de la aptitud física.

- Aptitud física (fitness o condición física): serie de atributos, como la resistencia, la flexibilidad y la fuerza, que se requieren para realizar actividades físicas.

- Deporte: actividad física organizada, que se rige por normas o reglas y se orienta a la competición.

En muchos países europeos, el término deporte abarca todo tipo de ejercicios $\mathrm{y}$ actividades físicas realizadas durante el tiempo libre.

- Actividad física moderada (aproximadamente, 3-6 MET $\left.^{*}\right)$ : requiere un esfuerzo moderado, que acelera de forma perceptible el ritmo cardíaco. Ejemplo: caminar a paso rápido; bailar; hacer jardinería o tareas domésticas; participar activamente en juegos libres y paseos con animales domésticos; desplazar cargas $<20 \mathrm{~kg}$.

- Actividad física intensa (aproximadamente, $>6 \mathrm{MET}^{*}$ ): requiere una gran cantidad de esfuerzo y provoca una respiración rápida y un aumento sustancial de la frecuencia cardíaca. Ejemplo: caminar ligero; ascender a paso rápido o trepar por una ladera; hacer desplazamientos rápidos en bicicleta, aerobic, natación rápida, deportes y juegos competitivos (por ejemplo, juegos tradicionales, fútbol, voleibol, hockey, baloncesto); realizar trabajos intensos con pala o excavación de zanjas; desplazar cargas pesadas (> $20 \mathrm{~kg})$.

* MET: unidad para expresar la intensidad de las actividades físicas denominada equivalente metabólico (MET). Un MET se define como el "costo energético de estar sentado tranquilamente" y es equivalente a un consumo de $1 \mathrm{kcal} / \mathrm{kg} / \mathrm{h}$. 


\section{BIBLIOGRAFÍA}

1. SAP: Comité Nacional de Medicina del Deporte Infantojuvenil, Comité de Medicina del Deporte Infantojuvenil (filial Córdoba). Consenso sobre Examen físico del niño y del adolescente que practica actividades físicas. Arch Argent Pediatr 2000;98(1):60-79.

2. SAP: Comité de Medicina del Deporte Infantojuvenil, Grupo de Trabajo de Salud Escolar, Comité de Cardiología, Comité de Adolescencia. Certificación para la actividad física escolar y pre-participativa deportiva. Arch Argent Pediatr 2001;99(6):538-41.

3. American Academy of Family Physicians, American Academy of Pediatrics, American College of Sports Medicine, American Medical Society for Sports Medicine, et al. PPE: Preparticipation Physical Evaluation. 4th ed. Illinois: American Academy of Pediatrics; 2010.

4. Vitiello R. Commentary: the value of the ECG in the preparticipation sports physicial examination: the Italian experience. Pediatr Rev 2006;27(11):e75-6.

5. Baptista CA, Foronda A, Baptista L de P. Esporte Competitivo na Criança e no Adolescente-Exame PréParticipação: Eletrocardiograma Obrigatório? Arq Bras Cardiol 2009;93(2):188-95.

6. Elliott J. Medical Conditions and Sports Participation. En: PreparticipationPhysicalEvaluationandMedicalClearance of Students Participating in Interscholastic Athletics and Select Auxiliary Units. Los Angeles unified school district Policy Bulletin October 1, 2010. Bul 4948;10-12.

7. Maron BJ, Thompson PD, Ackerman MJ, Balady G, et al. Recommendations and considerations related to preparticipation screening for cardiovascular abnormalities in competitive athletes: 2007 update: a scientific statement from the American Heart Association Council on Nutrition, Physical Activity, and Metabolism: endorsed by the American College of Cardiology Foundation. Circulation 2007;115(12):1643-55.

8. Maron BJ, Doerer JJ, Haas TS, Tierney DM, Mueller FO. Sudden deaths in young athletes: analysis of 1866 deaths in the United States, 1980-2006. Circulation 2009;119(8):108592.

9. Corrado D, Pelliccia A, Bjørnstad HH, Vanhees L, et al. Cardiovascular pre-participation screening of young competitive athletes for prevention of sudden death: proposal for a common European protocol. Consensus Statement of the Study Group of Sport Cardiology of the Working Group of Cardiac Rehabilitation and Exercise Physiology and the Working Group Myocardial and Pericardial Diseases of the European Society of Cardiology. Eur Heart J 2005;26(5):516-24.

10. PigozziF,SpataroA,FagnaniF,MaffulliN.Preparticipation Screening for the detection of cardiovascular abnormalities that may cause sudden death in competitive athletes. $\mathrm{Br}$ J Sports Med 2003;37(1):4-5.

11. Wever EFD, Robles de Medina EO. Sudden death in patients without structural heart disease. J Am Coll Cardiol 2004;43(7):1137-44.

12. Hong K, Brugada J, Oliva A, Berruezo-Sanchez A, et al. Value of electrocardiographicparameters and ajmaline test in the diagnosis of Brugada syndrome caused by SCN5A mutations. Circulation 2004;110(19):3023-7.

13. Pelliccia A, CorradoD. Can electrocardiographic screening prevent sudden death in athletes? Yes. BMJ 2010;341:c4923.

14. Wren C. Screening children with a family history of sudden cardiac death. Heart 2006;92(7):1001-6.

15. Joy EA, Paisley TS, Price R Jr, Rassner L, Thiese SM. Optimizing the collegiate preparticipation physical evaluation. Clin J Sport Med 2004;14(3):183-7.

16. Singh A, Silberbach M. Consultation with the specialist: cardiovascular prepaticipation sports screening. Pediatr Rev 2006;27(11):418-24.

17. Sofi F, Capalbo A, Pucci N, Giuliattini J, et al. Cardiovascular evaluation, including resting and exercise electrocardiography, before participation in competitive sports: cross sectional study. BMJ 2008;337:a346.

18. Baggish AL, Hutter AM Jr, Wang F, Yared K, et al. Cardiovascular screening in college athletes with and without electrocardiography: A cross-sectional study. Ann Intern Med 2010;152(5):269-75.

19. Le VV, Wheeler MT, Mandic S, Dewey F, et al. Addition of the electrocardiogram to the preparticipation examination of college athletes. Clin J Sport Med 2010;20(2):98-105.

20. Corrado D, Basso C, Pavei A, Michieli P, et al. Trends in sudden cardiovascular death in young competitive athletes after implementation of a preparticipation screening program. JAMA 2006;296(13):1593-601.

21. Garrick JG. Preparticipation orthopedic screening evaluation. Clin J Sport Med 2004;14(3):123-6.

22. Behm D, Faigenbaum AD, Falk B, Klentrou P. Canadian Society for Exercise Physiology position paper: resistance training in children and adolescents. Appl Physiol Nutr Metab 2008;33(3):547-61.

23. Rice SG. Medical conditions affecting sports participation. Pediatrics 2008;121(4):841-8.

24. American Academy of Family Physicians, American Academy of Pediatrics, American College of Sports Medicine, American Medical Society for Sports Medicine, et al. Preparticipation Physical Evaluation. 3rd ed. New York: McGraw-Hill; 2004.

25. Maron BJ, Zipes DP. Introduction: elegibility recommendations for competitive in athletes with cardiovascular abnormalities. I Am Coll Cardiol 2005;45(8):1318-21. 


\section{ANEXO 1}

\section{MODELO DE FICHA MÉDICA (Anamnesis)}

Apellido y nombre:

Fecha de nacimiento: / /

Domicilio:

Localidad:

Teléfono 1:
DNI:

Edad: Sexo:

$\mathrm{N}^{\circ}$ : $\quad$ Piso

Provincia: $\quad \mathrm{CP}$

Teléfono 2: Correo elctrónico:

La siguiente información es de suma importancia para el profesional que lo atiende. Durante el examen físico se evaluará el estado clínico actual.

ANTECEDENTES PERSONALES. Marque lo que corresponda.

En menores de 14 años, deberá ser completado obligatoriamente por el padre, la madre o el responsable legal.

En adolescentes de entre 14 y 18 años se recomienda que sea completado por el padre, la madre o el responsable legal.

En caso que el adolescente concurra solo a la consulta podrá completarla él mismo si conoce los antecedentes.

\begin{tabular}{|c|c|c|c|c|c|}
\hline & Sí & No & & Sí & No \\
\hline Problemas cardíacos & & & Afecciones auditivas & & \\
\hline Presión arterial elevada & & & Fracturas, luxaciones, lesiones ligamentarias & & \\
\hline Obesidad & & & Otro problema en los huesos o articulaciones & & \\
\hline Asma bronquial & & & Cirugías & & \\
\hline Pérdida de conciencia & & & Internaciones & & \\
\hline Convulsiones & & & Enfermedades infecciosas en los últimos 2 meses & & \\
\hline Traumatismos de cráneo & & & $\begin{array}{l}\text { Falta o no funcionamiento de algún órgano par: } \\
\text { riñón, ojos, testículo }\end{array}$ & & \\
\hline Diabetes & & & Alergias & & \\
\hline Alteraciones sanguíneas & & & Tos crónica & & \\
\hline \multirow{6}{*}{$\begin{array}{l}\text { En relación con el ejercicio (durante } \\
\text { o después), ha padecido alguna vez: } \\
\text { Desmayos } \\
\text { Mareos } \\
\text { Dolor fuerte en el pecho } \\
\text { Mayor cansancio que sus compañeros }\end{array}$} & & & Medicación habitual & & \\
\hline & & & Alergia a algún medicamento & & \\
\hline & & & Consume vitaminas & & \\
\hline & & & Problemas de piel & & \\
\hline & & & Usa anteojos & & \\
\hline & & & & & \\
\hline Palpitaciones & & & & & \\
\hline $\begin{array}{l}\text { Dificultad para respirar durante o } \\
\text { después de la actividad física }\end{array}$ & & & & & \\
\hline Vacunación completa & & & & & \\
\hline
\end{tabular}

Observaciones (detalle las respuestas afirmativas):

Antecedentes familiares

\begin{tabular}{|l|l|l|l|l|}
\hline $\begin{array}{l}\text { Muerte súbita en familiar } \\
\text { directo menor de } 50 \text { años }\end{array}$ & & Obesidad & & \\
\hline & & Diabetes & \\
\hline Problemas cardíacos & & & Colesterol elevado & \\
\hline Presión arterial elevada & & & Tos crónica & \\
\hline
\end{tabular}

Presión arterial elevada

Observaciones:

Por la presente afirmo que, dentro de mis conocimientos y de acuerdo con las preguntas planteadas mis respuestas son veraces.

Fecha:

Firma del padre, la madre

o el responsable legal
Firma del adolescente

(14 a 18 años) 


\section{EXAMEN FÍSICO}

\section{ANEXO 2}

\section{Anamnesis personalizada:}

En relación con el ejercicio (durante o después), ha padecido alguna vez:

Desmayos:

Mareos:

Dolor fuerte en el pecho:

Mayor cansancio que sus compañeros:

Palpitaciones:

Dificultad para respirar durante o después de la actividad física:

Consumo de vitaminas, minerales, bebidas energizantes, suplementos:

Consumo de alcohol, cigarrillos, otras sustancias:

\begin{tabular}{|c|c|c|c|c|}
\hline $\operatorname{Pc}()$ & Talla: & \multirow{2}{*}{\multicolumn{2}{|c|}{$\begin{array}{l}\text { Pc ( ) } \\
\text { Ortodoncia fija: sí/no } \\
\text { Oclusión dental: }\end{array}$}} & \multirow[t]{2}{*}{$\operatorname{Pc}()$} \\
\hline Examen bucodental: & Caries: sí/no & & & \\
\hline Examen visual: & OD: & \multicolumn{2}{|l|}{ OI: } & $\begin{array}{l}\text { Pupilas: } \\
\text { Visión cromática: }\end{array}$ \\
\hline Examen cardiovascular & $\begin{array}{l}\text { Inspección: } \\
\text { Auscultación: } \\
\text { FC: } \\
\text { TA: } \\
\text { Pulsos humerales: } \\
\text { Pulsos femorales: }\end{array}$ & $\operatorname{Pc}()$ & & \\
\hline Examen respiratorio & \multicolumn{2}{|l|}{ Inspección: } & \multicolumn{2}{|l|}{ Auscultación: } \\
\hline Examen abdominogenital & \multicolumn{2}{|l|}{ Inspección: } & \multicolumn{2}{|l|}{ Palpación: } \\
\hline Examen músculo esquelético & Actitud & \multicolumn{2}{|l|}{ Postura } & Asimetrías \\
\hline \multicolumn{2}{|c|}{ Movilidad articular } & \multicolumn{2}{|l|}{ Cuello } & Tronco \\
\hline \multicolumn{2}{|c|}{ Apoyo plantar } & \multicolumn{2}{|c|}{ Extremidades superiores } & Extremidades inferiores \\
\hline \multicolumn{2}{|l|}{ Evaluación madurativa } & \multicolumn{2}{|l|}{ Menarca: } & Ritmo menstrual: \\
\hline \multicolumn{5}{|c|}{ Observaciones: (detallar hallazgos) } \\
\hline \multicolumn{5}{|l|}{ Indicaciones: } \\
\hline \multirow[t]{2}{*}{ Lugar y fecha: } & & \multicolumn{3}{|c|}{ Firma y sello del médico } \\
\hline & & $\begin{array}{l}\text { Aclar } \\
\mathrm{N}^{\circ} \text { de }\end{array}$ & $\begin{array}{l}\text { ón: } \\
\text { atrícula: }\end{array}$ & \\
\hline
\end{tabular}




\section{ANEXO 3}

\section{PATOLOGÍAS MÉDICAS Y PARTICIPACIÓN DEPORTIVA (American Academy of Pediatrics, 2008) ${ }^{23}$}

Patología

Participa

Inestabilidad atlantoaxial (inestabilidad de la articulación entre la primera y la segunda vértebra cervical)

Explicación: el atleta (sobre todo si padece síndrome de Down o artritis reumatoide juvenil con afectación cervical) necesita una evaluación para determinar el riesgo de lesión de la médula espinal durante la participación en el deporte, especialmente al utilizar el trampolín.

Trastorno hemorrágico

Explicación: el atleta necesita evaluación.

Enfermedad cardiovascular: carditis

Explicación: puede resultar en la muerte repentina con el esfuerzo.

Hipertensión (tensión arterial elevada).

Explicación: los que tienen una hipertensión $>5 \mathrm{~mm} \mathrm{Hg}$ por encima del percentil 99 para la edad, el sexo

y el percentil de talla deberían evitar la halterofilia, el culturismo y los deportes con un gran componente estático.

Los que tienen hipertensión sostenida (> 95 percentil para la edad, el sexo y el percentil de talla) necesitan evaluación.

Cardiopatía congénita

Explicación: se recomienda la consulta con un cardiólogo. Los que presentan formas leves pueden participar por completo en la mayoría de los casos; los afectados en formas moderadas o graves o los sometidos a cirugía necesitan evaluación. La $36^{\circ}$ Conferencia de Bethesda definió la enfermedad leve, moderada y grave de las lesiones cardíacas comunes.

Alteración del ritmo cardíaco

Síndrome de QT prolongado

Arritmias ventriculares malignas

Síndrome de Wolff-Parkinson-White sintomático

Bloqueo cardíaco avanzado

Antecedente familiar de muerte repentina o ataque cardíaco repentino previo

Implante de un cardioversor-desfibrilador

Explicación: se aconseja la consulta con un cardiólogo. Los que presentan síntomas (dolor torácico, síncope, amenaza de síncope, mareos, respiración entrecortada u otros síntomas de posibles alteraciones del ritmo) o pruebas de insuficiencia mitral en la exploración física necesitan evaluación. Los demás pueden participar sin limitaciones.

Soplo cardíaco

Explicación: si el soplo es inocente, se permite la participación sin limitaciones.

En otro caso, el atleta necesita una evaluación.

Cardiopatía estructural/adquirida

- Miocardiopatía hipertrófica

- Anomalías de las arterias coronarias

- Miocardiopatía arritmogénica del ventrículo derecho

- Fiebre reumática aguda con carditis

- Síndrome de Ehlers-Danlos, forma vascular

- Síndrome de Marfan

- Prolapso de la válvula mitral

- Empleo de antraciclina

Explicación: se recomienda la consulta con un cardiólogo. La $36^{\circ}$ Conferencia de Bethesda ofreció

recomendaciones detalladas. La mayoría de estas alteraciones comporta un riesgo importante de muerte cardíaca repentina asociada con el ejercicio físico intenso. La miocardiopatía hipertrófica requiere un meticuloso trabajo diagnóstico y reiteradas valoraciones, porque la enfermedad puede cambiar sus manifestaciones al final de la adolescencia. El síndrome de Marfan con aneurisma aórtico también puede causar la muerte repentina durante el ejercicio físico intenso. El atleta que haya recibido en alguna ocasión quimioterapia con antraciclinas puede correr mayor riesgo de padecer problemas cardíacos por los efectos cardiotóxicos de los medicamentos, y el entrenamiento de resistencia en esta población debe abordarse con precaución.

Vasculitis/enfermedad vascular calificada

- Enfermedad de Kawasaki (vasculitis de la arteria coronaria)

- Hipertensión pulmonar

Explicación: se recomienda la consulta con un cardiólogo. El atleta necesita la valoración individual para estimar el riesgo según la actividad de la enfermedad, las alteraciones patológicas y el régimen médico.

Parálisis cerebral 
Explicación: el atleta necesita una evaluación para determinar la capacidad funcional para realizar la actividad deportiva específica.

Diabetes mellitus

Explicación: se puede practicar cualquier deporte con la adecuada atención al ajuste de la dieta (especialmente, la ingestión de hidratos de carbono), la glucemia, la hidratación y el tratamiento con insulina. Se debe controlar la glucemia antes del ejercicio, cada 30 min durante el ejercicio sostenido, 15 min después de finalizar el ejercicio $\mathrm{y}$ al acostarse.

Diarrea infecciosa

Explicación: a menos que los síntomas sean leves y que el atleta esté totalmente hidratado, no se permite la participación porque la diarrea puede aumentar el riesgo de deshidratación y de golpe de calor.

Trastornos de la alimentación

Explicación: el atleta con trastorno de la alimentación necesita de una valoración médica y

psiquiátrica antes de participar.

Ojos

Atleta funcionalmente tuerto

Pérdida de un ojo

Desprendimiento de retina o antecedente familiar de desprendimiento de retina a edad joven

Miopía intensa

Trastorno del tejido conjuntivo, como síndrome de Marfan o de Stickler

Cirugía intraocular o lesión ocular grave previa

Explicación: el atleta funcionalmente tuerto se define como el que tiene una mejor agudeza visual corregida inferior a 20/40 en el ojo de peor visión. Este atleta padecería una discapacidad importante si el ojo de mejor visión resultase gravemente lesionado, igual que el atleta con pérdida de un ojo. Específicamente el boxeo y las artes marciales de pleno contacto están contraindicados para los atletas funcionalmente tuertos, porque la protección ocular es imposible o no está autorizada. Algunos atletas sometidos previamente a cirugía intraocular o con una lesión ocular grave pueden correr mayor riesgo de lesión por el debilitamiento del tejido ocular. La disponibilidad de protectores oculares y de otro equipamiento protector puede permitir la participación en la mayoría de los deportes, aunque se debe juzgar de forma individual.

Conjuntivitis infecciosa

Explicación: el atleta con conjuntivitis infecciosa activa debe ser excluido de la natación.

Fiebre

Explicación: la elevada temperatura interna puede indicar una alteración médica patológica

(infección o enfermedad) que, a menudo, se manifiesta por un aumento del metabolismo y la frecuencia

cardíaca en reposo. Por consiguiente, durante el régimen de ejercicio habitual del atleta, la presencia de fiebre puede resultar en una mayor conservación de calor, una disminución de la tolerancia al calor, un aumento del riesgo de golpe de calor, un aumento del esfuerzo cardiopulmonar, una disminución de la capacidad máxima de esfuerzo y un aumento del riesgo de hipotensión debido a la alteración del tono vascular y la deshidratación. Además, en raras ocasiones, la fiebre puede acompañar a la miocarditis o a otras alteraciones que pueden hacer peligroso el ejercicio habitual.

Gastrointestinal

Síndromes de malabsorción (enfermedad celíaca, fibrosis quística)

Explicación: el atleta necesita de la valoración individual respecto a la malnutrición general o a las deficiencias específicas resultantes en defectos de la coagulación o de otro tipo; con el tratamiento adecuado, estas deficiencias pueden ser tratadas adecuadamente para permitir las actividades normales.

Síndrome del intestino corto $\mathrm{u}$ otros trastornos que necesiten apoyo nutricional especializado, que incluyen la nutrición parenteral o enteral

Explicación: el atleta necesita la valoración individual para los deportes de colisión, contacto o contacto limitado.

Antecedente de golpe de calor

Explicación: dada la probabilidad de recurrencia, el atleta necesita una evaluación individual para determinar la presencia de alteraciones y comportamientos predisponentes, y para desarrollar una estrategia de prevención que incluya la suficiente aclimatación (tanto al ambiente como a la intensidad y la duración del ejercicio), el entrenamiento, la hidratación y la ingestión de sal, así como otras medidas eficaces para mejorar la tolerancia al calor y disminuir el riesgo de golpe de calor (como equipamiento protector y configuraciones del uniforme).

Hepatitis infecciosa (principalmente la hepatitis C)

Explicación: todos los atletas deben haber recibido la vacuna contra la hepatitis B antes de participar.

Dado el mínimo riesgo aparente para los demás, el atleta puede practicar todos los deportes que le permita su estado de salud.

Se deben cubrir adecuadamente las lesiones cutáneas de todos los atletas, y el personal atlético debería adoptar precauciones universales al manejar sangre y líquidos orgánicos con sangre visible.

Infección por el virus de la inmunodeficiencia humana

Explicación: dado el mínimo riesgo aparente para los demás, el atleta puede practicar todos los deportes que 
adecuadamente las lesiones cutáneas de todos los atletas, y el personal atlético debería adoptar precauciones universales al manejar sangre y líquidos orgánicos con sangre visible. Sin embargo, determinados deportes (como la lucha libre o el boxeo) pueden crear una situación favorable para la transmisión del virus (como el sangrado más la rotura de la piel). Si la carga vírica es detectable, se debe aconsejar a estos atletas que eviten los deportes de contacto intenso.

Ausencia de un riñón

Explicación: los atletas necesitan la evaluación individual para los deportes de contacto, colisión y contacto limitado. El equipamiento protector puede disminuir el riesgo de lesión del riñón restante lo suficiente como para permitir la participación en la mayoría de los deportes, siempre que este equipamiento siga colocado durante la actividad.

Hepatomegalia/esplenomegalia

Explicación: si el tamaño del hígado aumenta bruscamente, se debe evitar la participación por el riesgo de rotura. Si el aumento de tamaño es crónico, es necesaria la evaluación individual antes de practicar deportes de colisión, contacto o contacto limitado. Los pacientes con hepatopatía crónica pueden tener alteraciones de la función hepática, que pueden afectar el vigor, el estado intelectual, la coagulación o el estado nutricional.

Neoplasia maligna

Explicación: el atleta necesita una evaluación individual.

Trastornos musculares

Explicación: el atleta necesita una evaluación individual.

Trastornos neurológicos

Antecedente de traumatismo o anomalía craneal o vertebral grave, que incluye craneotomía, sangrado epidural, hematoma subdural, hemorragia intracerebral, síndrome del segundo impacto, malformación vascular y fractura de cuello

Explicación: el atleta necesita una evaluación individual para practicar deportes de colisión, contacto o contacto limitado.

Antecedente de concusión simple (lesión traumática cerebral leve), concusiones simples múltiples, concusión compleja o una combinación de ellas

Explicación: el atleta necesita una evaluación individual. La investigación apoya el abordaje conservador del tratamiento de la concusión, que incluye la ausencia de participación deportiva mientras presenta síntomas o cuando se detectan deficiencias del juicio o la cognición, seguido de un retorno gradual y secuencial a la actividad completa.

Miopatías

Explicación: el atleta necesita una evaluación individual.

Cefaleas recurrentes

Explicación: el atleta necesita una evaluación individual.

Plexopatía recurrente (quemazón o picor) y neuropraxia de la médula cervical con defectos persistentes Explicación: el atleta necesita una evaluación individual para practicar deportes de colisión, contacto o contacto limitado; recuperar la fuerza normal es un punto de referencia importante para volver a jugar.

Trastorno convulsivo, bien controlado

Explicación: el riesgo de convulsión durante la participación es mínimo.

Trastorno convulsivo mal controlado

Explicación: el atleta necesita una evaluación individual para practicar deportes de colisión, contacto o contacto limitado.

Se deben evitar los siguientes deportes sin contacto: tiro con arco, tiro al blanco, natación, halterofilia, entrenamiento de fuerza o deportes que impliquen alturas. En estos deportes, la aparición de una convulsión durante la actividad puede imponer un riesgo a sí mismo o a los demás.

Obesidad

Explicación: dado el mayor riesgo de golpe de calor y de tensión cardiovascular, el atleta obeso necesita particularmente de una meticulosa aclimatación (tanto al ambiente como a la intensidad y la duración del ejercicio), una hidratación suficiente y la posible modificación de la actividad y la recuperación durante la competición y el entrenamiento.

Receptor de trasplante (y los que toman medicamentos inmunodepresores)

Explicación: el atleta necesita una evaluación individual para practicar deportes de contacto, colisión y contacto limitado.

Además del posible riesgo de infecciones, algunos medicamentos (como la prednisona) pueden aumentar la tendencia a la formación de hematomas.

Ausencia de ovario

Explicación: el riesgo de lesión grave del ovario restante es mínimo.

Embarazo/puerperio

Explicación: el atleta necesita una evaluación individual. A medida que progresa el embarazo, será necesario modificar las rutinas habituales de ejercicio. Se deben evitar las actividades con gran riesgo de caída o de traumatismo abdominal. También se debe evitar durante el embarazo el buceo y las actividades que impongan un riesgo de vértigo. 
Tras el parto, los cambios fisiológicos y morfológicos del embarazo tardan de 4 a 6 semanas en regresar a la situación inicial.

Alteraciones respiratorias

Compromiso pulmonar, que incluye la fibrosis quística

Explicación: el atleta necesita una evaluación individual, pero, en general, puede practicar cualquier deporte si la oxigenación es satisfactoria durante una prueba de esfuerzo gradual. Los atletas con fibrosis quística necesitan aclimatación y buena hidratación para disminuir el riesgo de golpe de calor.

Asma

Explicación: con los medicamentos y la formación oportuna, solo los atletas con el asma más grave deberán modificar su participación. Se debe recomendar un plan de actuación escrito y el empleo diario de un medidor del flujo máximo a los que utilizan inhaladores. Los atletas con asma pueden tener problemas durante el buceo.

Infección aguda de las vías respiratorias altas

Explicación: la obstrucción respiratoria alta puede alterar la función pulmonar. El atleta necesita una evaluación individual en todos los grados de enfermedad, menos los leves.

Enfermedades reumatológicas

Artritis reumatoide juvenil

Explicación: los atletas con artritis reumatoide juvenil sistémica o poliarticular y antecedente de afectación de la columna cervical necesitan someterse a radiografías de las vértebras C1-C2 para evaluar el riesgo de lesión de la médula espinal. Los atletas con artritis sistémica o asociada con HLA B27 necesitan una evaluación cardiovascular de las posibles complicaciones cardiovasculares durante el esfuerzo. Los protectores bucales son útiles para quienes presentan micrognatia (mordida abierta y dientes expuestos). Si existe uveítis, el riesgo de lesión ocular traumática aumenta; se recomienda la evaluación oftalmológica y, si existe alteración visual, se deben seguir las pautas para los atletas funcionalmente tuertos.

Dermatomiositis juvenil (DMJ), miositis idiopática

Lupus eritematoso sistémico (LES)

Fenómeno de Raynaud

Explicación: el atleta con DMJ o LES y afectación cardíaca necesita de una evaluación cardiológica antes de la participación. Los atletas que reciben corticoides sistémicos corren mayor riesgo de fracturas osteoporóticas y de necrosis avascular, que se debe evaluar antes de su eliminación; los que reciben medicamentos inmunodepresores corren mayor riesgo de infección grave. Se debe evitar la actividad deportiva mientras la miositis esté activa.

La rabdomiolisis durante el ejercicio intenso puede causar una lesión renal a los atletas con miositis idiopática y otras miopatías. Dada la fotosensibilidad de DMJ y LES, es necesaria la protección solar durante la actividad al aire libre. En el fenómeno de Raynaud, la exposición al frío impone riesgo en las manos y los pies.

Infecciones de la piel: incluyen herpes simple, molusco contagioso, verrugas, infecciones estafilocócicas

y estreptocócicas (forúnculos, ántrax, impétigo, Staphylococcus aureus resistente a meticilina -celulitis,

absceso, o ambos-), sarna, tiña

Explicación: durante el período contagioso, no se permite la participación en gimnasia o animación con compañeros, las artes marciales, la lucha libre u otros deportes de colisión, contacto o contacto limitado.

Testículo no descendido o ausencia de uno

Explicación: determinados deportes pueden necesitar de un protector. 